\title{
Contemporary Geopolitics and Digital Representations of Space
}

Sead Turčalo, Ado Kulović

\section{Abstract}

This research is premised on two theoretical constructs: that maps do not objectively depict space and that traditional cartography produces a geopolitical narrative. The research aim is to investigate geopolitical influence in modern, digital representations of space, and vice versa. This paper is divided into three parts: In the first, the digital representation of space is introduced and explained, and two widely acknowledged digital cartographic services are established as the empirical foundation of the research - Google (Google Maps and Google Earth), designed by cartographic and geo-data professionals, and OpenStreetMap, built through crowdsourcing. In the second part, the geopolitical features of traditional cartography are discussed in the context of digital mapping, including ethnocentricity and hierarchical representations of space, similarities to geopolitische karte, and "minor geopolitics." The final part asks and answers a key question about geopolitical subjectivity: "Who benefits from the geopolitical narratives in digital representations of space?"

\section{KEY WORDS:}

geopolitics, Geoweb, cartography, Google Maps and Google Earth, OpenStreetMap (OSM) 


\section{Introduction}

Since the 1890s, the term 'geopolitics' has "provoked personal passion and intellectual outbursts" (Dodds and Atkinson 2003: xiv). Yet to this day, the term lacks a generally understood meaning. Nevertheless, nearly all authors accept that the most significant ontological element of geopolitics is space. Cartography, the main means by which space is depicted, is thus inherently linked to geopolitics. However, maps are not objective representations of space. As Yves Lacoste has noted, the map is a powerful tool, which abstracts concrete reality based on practical concerns and "facilitates domination and control" (Dodds 2007: 120). Hence, it is safe to assert that geopolitical narratives exist within cartography.

Modern, computer-aided cartography is introducing previously unknown ways to represent space and is offering greater accessibility and new roles for users of digital maps. At the same time, geopolitical theory and practice have also evolved. It is in the context of these multiple paradigm changes that we saw an opportunity for scientific inquiry and tasked ourselves with examining geopolitical narratives within modern depictions of space. In other words, we sought to understand the connection between contemporary geopolitics and representations of space on digital platforms.

This was, as it turned out, a difficult undertaking. The research process was burdened by the lack of consensus on a definition of geopolitics and an absence of any previous scientific research on this topic. Thus, we had to develop our own research and methodological approaches. To this end, we identified three central research questions: Firstly, what is a digital representation of space and what are its characteristics? Secondly, what are the geopolitical properties of a digital representation of space? And thirdly, who benefits from the geopolitical narratives reflected in digital representations of space?

In line with this methodological approach, this paper is divided into three parts. The first discusses the development of computer-aided cartography services and introduces the concept of the "Geoweb." The second examines the geopolitical properties of (mostly) traditional maps, 
but in the digital context, to determine how the geopolitical narratives of conventional cartography influence the development of digital mapping, and how "new spatial media" is changing geopolitical practice and theory. The final part deals with the geopolitical narrative of the Geoweb and tries to ascertain which geopolitical agents it benefits.

\section{Digital representations of space: the Geoweb}

The digital representation of space relies on the descriptive display of space and the technology that facilitates it. Crampton calls it "new spatial media" (2009a: 92) but acknowledges that a bewildering variety of terms have been applied to the topic'. Citing Wood (2003), he notes that, "ironically 'cartography' does not seem to be" among them (2009a: 91). We have concluded that the term Geoweb is generally accepted by most authors. In introducing the term here, we feel it is helpful to explain the origins of the two technologies without which the Geoweb would not exist: the Geographical Information System (GIS) and remote-sensing space technology.

Digital cartography is not as new as one may assume. Farman explains that its conception dates back to the 1950s, "often in conjunction with the mapping of census data and land use," and that the first ever GIS was the "Canada Geographic Information System," launched in 1964 (2010: 870). In 1982, an open source variant of GIS (GRASS) was conceived (Crampton 2009a: 94); and by the end of the 1980s, computer-aided cartography and GIS were common within the cartographic community. Indeed, in the late 1980s, Harley commented that the embrace of computer-aided technology was causing map makers to become "more strident" and concluded that a "culture of technics" was widespread (1989: 2).

Remote-sensing space technology was, unsurprisingly, a cold war-era innovation. It began with three American imaging satellite programmes

"...geospatial web or geoweb (Scharl and Tochtermann, 2007), neogeography (Turner, 2006), locative media (Rheingold, 2002), DigiPlace (Zook and Graham, 2007a), spatial crowdsourcing or geocollaboration (Hopfer and MacEachren, 2007) and map hacking (Erle et al., 2005)" (Crampton 2009a: 91). 
(dubbed Corona, Argon, and Lanyard), which were active from 1959 to 1972 and were operated by the US Central Intelligence Agency. These 'photographic surveillance satellites' gathered data to assess Soviet longrange bomber development. But this "worldwide photographic coverage was also used to produce maps and charts for the Department of Defense and other US government mapping programs" (Program Corona, Mission and Spacecraft Library - NASA JPL) ${ }^{2}$. In 1972, NASA initiated "Landsat" the first civilian remote-sensing satellite programme. A private firm, Earth Observation Satellite Company (EOSAT), began operating Landsat in 1985; but one year later, the programme's commercial monopoly on Earth imaging ended after the French SPOT satellite was launched. In 1992, NASA took back Landsat for itself, though its prominence has been diminished by the emergence in this field of other governments as well as private companies (Lee 2010: 918).

The first popular online map service was MapQuest, launched in 1996 (Farman 2010: 870; Parsons 2013: 182). Parsons asserts that, although similar online services existed (e.g. Xerox Parc View, started in 1993), MapQuest gained attention through the service it offered to non-academic web users. The next step in online map development, he explains, were consumer-focused services such as store finders (2013: 182).

In 2004, the most successful crowdsourced mapping project OpenStreetMap (OSM) - was introduced, offering the facility of a free, open-source global map (Caquard 2011: 137). According to Gerlach, "OpenStreetMap is at once a technology, a set of performances and a series of communities that allow users to create and alter maps, based on wiki protocols" (2015: 276). This represents so-called citizen cartography or "Volunteered Geographic Information" (VGI) (Goodchild 2007 by Parsons 2013: 182). As of August 2017, OSM had some 4.3 million registered users (Stats OpenStreetMap wiki). The major share of these are male and more than half have formal training in GIS (Budhathoki 2010 by Gerlach 2015: 276).

The most-accessed new spatial media are Google's Maps and Earth services. Started in February 2005, Google Maps initially utilised

2 The Program's existence was not revealed until 1995 by President Clinton's Executive Order (Program Corona, Mission and Spacecraft Library - NASA JPL). 
MapQuest's service to support local searches. Today, it relies on the remote-sensing images that are the core of Google Earth (Parsons 2013: 182). It is important to note that, contrary to popular belief, Google does not own any satellites. It obtains images from third-party providers such as the aforementioned Landsat.

The Geoweb is short for "Geospatial Web," which Offen described as a combination of applications and new skills that search for georeference information throughout the web (2012: 567). According to Crampton, free and open source software (FOSS) is a significant philosophical element of the Geoweb; and one which divides new spatial media and GIS into two camps (2009a: 93, 97) - on the one hand, the FOSS Geoweb, and on the other, efforts to accredit mapping expertise through professional certification and "bodies of knowledge" (DiBiase et al. 2006; Crampton 2009a: 97). This paper focuses on OSM and Google, the most prominent Geoweb services. The former is an example of the FOSS Geoweb and the latter of the institutionalized, "professional" Geoweb. On these platforms, we will analyse the connection between digital representations of space and contemporary geopolitical practice and theory. We are, therefore, viewing these platforms as the empirical foundation for this paper's theoretical argumentation.

\section{Geopolitical properties}

In Agnew's discussion of visualizing global space, he explains that two perspectives "emerged at the outset of the European Age of Discovery;" one of which positions the world as "an ordered, structured whole," that is separate from and outside of the viewer (2003: 15). Given that the distance between a viewer of a global map and the world itself can be observed as a geopolitical manifestation, and since the Geoweb is, in ontological terms, the "world-as-a-picture," the geopolitical properties of this map must be examined. Harley explains that there are two sets of cartographic properties, related to their cultural production and their technical production (Harley 1989: 6); or, as Agnew describes it, two features of maps: ethnocentricity, which adds geopolitical meaning 
through "a kind of subliminal geometry," and hierarchical representation, which identifies and names sites in social terms (Agnew 2003: 19). Both of these features are present in the Google Geoweb ${ }^{3}$ and in OSM.

Regarding ethnocentricity, Agnew notes that global maps are most often "centred on Europe," and that the Mercator projection depicts "a clear sense of Europe's geopolitical centrality" (2003: 19). This is the case with Google and OSM maps, which are based on the EPSG:3587 Spherical Mercator projection (EPSG: 3857 OpenStreetMap wiki). Hierarchical representation is evident in more than one property of Geoweb base maps, perhaps most obviously in a proportional emphasis on spaces related to their perceived importance (Harley 1989: 7). Geoweb base maps of Western countries are developed first and, generally, more thoroughly4; though this is less evident in OSM maps, which are not developed by a single entity. Hierarchical representation of space can also manifest in how sites are named and identified, as disputed sites reveal. Two examples from the Google Geoweb are the delineation of Kosovo's border, shown by a dashed line in Google Maps and a thin red line in Google Earth; and the labelling of the Falkland Islands, which prioritises the British name and subordinates the Argentinian name, Islas Malvinas, in parentheses. It should be noted that, in OSM, the labelling of disputed areas can be the subject of constant change ${ }^{5}$.

Dodds argues that public interest in maps is greatest during times of war and global tension (2007: 115). Yet, we contend that the Geoweb somewhat changes how maps are consumed as well as the geopolitical properties of the add-ons applied to base maps. Before the Geoweb, traditional (printed) maps were more difficult to access than new spatial media. If a consumer needed a map, they had to buy it, borrow it from a library, or receive it in some kind of public distribution (e.g., as propaganda). In other words, a special effort had to be made by either the consumer or creator, which makes it understandable that public interest in maps heightened at specific times. The convenience of the Geoweb is paradigm changing. Although consumers are still more prone to use maps when needed for a particular purpose, in the Geoweb, this

3 The term "Google Geoweb" is used here to collectively describe the Google Maps and Google Earth services.

4 This is clear in comparing the Street View option in the Google Geoweb in places in Europe and North America with places in developing countries.

5 This will be expounded upon in the next part of the paper. 
"need" may be more trivial. To use the Geoweb, a consumer requires only an Internet connection and an adequate device. Thus, for a "need" to emerge, they must only be curious; no international crisis or prospect of war must exist. Of course, the Internet and concomitant devices are not equally accessible everywhere. The paradigm shift ushered in by the Geoweb is thus constrained by the affordability of technology.

There is also the question of cartographic participation in new spatial media. It is not hard to recognise similarities between the Google Geoweb and the geopolitische karte (geopolitical maps) introduced in 1920s Italy and Germany. Boria has written an extensive paper about this controversial type of cartography, noting that "these maps represent a genuine leap forward, an added sophistication in cartographic communication techniques.... [W] hile traditional cartography presents few political elements (e. g. borders, capitals) and portrays a static political situation, a geopolitical map renders the picture dynamic, showing the historical causes of a given political situation, possible future developments thereof, or both" (2008: 280). Designed to be used by the public at large, geopolitical maps are accessible even to "many who are unfamiliar with traditional geographical maps" (Boria 2008: 280).

The main technical design innovation of geopolitical maps was the use of geometric shapes "to represent factors affecting the organization of political space" (Boria 2008: 281) ${ }^{6}$. As Gordon has pointed out, almost instantly upon Google's release of its Geoweb, users began developing APIs (application programming interfaces) to create 'mashups' from existing data; and in July 2005, Google released its own API, free of charge (2007: 894). In a general sense, a mashup, or overlay, represents an API that combines geographical data from one source with a map from another (Butler 2006a; Miller 2006 and Anon 2007 by Crampton 2009a: 93). In the case of the Google Geoweb, the aim is to transform maps into programmable objects (Plantin et al. 2016: 13). This amounts to the literal replacement of existing maps (Farman 2010: 873). And put simply, this means that anyone can create a spatial narrative, tell a story, suggest an idea or a theory, promote an ideology, or conduct geopolitical activities

6 Meaning, "...arrows to indicate territorial conquest or commercial penetration, axes for alliance systems, circles or half-circles for spheres of influence, parallel lines to mark equivalent or reciprocal tendencies, broken lines for uncertainty, radial and linear structures, interrupted lines as a sign of disintegration, stars and diamonds to indicate the hubs of political forces in action, as well as borders and shadings in abundance - all are graphic solutions typical of geopolitical cartography." 
by employing symbols on a map. The parallels to geopolitische karte are apparent, with a key difference - the geopolitical dimension in new spatial media is executed by a wide range of independent participants, instead of by an official institution or individual.

There are many types of mashups. The Google Maps Mania blog alone offers about 8,500 for download. These mashups can be browsed by date of upload and each one features a description, which makes it easy to place a mashup in context. Take, for example, a mashup named "Why NATO isn't obsolete," created in mid-January 2017, shortly after US President Donald Trump used this term to describe the Alliance. The mashup features a "security challenges" layer, which the creator says "shows some of the present global threats to peace and security that NATO and its partners currently faces [sic] across the globe" (Why NATO isn't Obsolete, Google Maps Mania). This mashup also offers an interactive view, in which civil and military capabilities, missile ranges, and NATO members, allies, and friends are presented as geometric shapes. It is significant that this mashup was created after the provocative and rather geopolitical statement of a very powerful world leader, and its creation illustrates how mashups are often inspired by recent events. Indeed, the more dramatic an event, the more Google Geoweb mashups (in volume and elaboration) follow.

So, does this substantiate the claim of Dodds that popular demand for maps rises in times of turbulence? It seems the Geoweb generally follows this pattern, but in terms of participation not consumption. "Interest has shifted from the map as an object to mapping as practice.... [and] some authors suggest that cartography be understood as existence (becoming) rather than essence (fixed ontology)" (Crampton 2009b: 840; emphasis in original). Participation in the Geoweb has its own geopolitical dimensions as well. As noted, in the Google Geoweb, participation is limited to addon creation. However, in OSM, participation lies at the core of the project.

While accepting that mapping is a geopolitical process, as far as "its implication in the practices of statecraft and of delineating sovereignty in a 'major' representational sense," Gerlach discusses the notion of a "minor geopolitics" related to participatory mapping. In his use, the term minor is not conflated with size or scale but with the depiction of non- 
representational features, as a way of exposing the unspoken politics of mapping. In that regard, mapping "is also geopolitical in the way in which it cultivates affects, attitudes, bodily dispositions, collectives, sensibilities, spaces and events that are transformative of the world, but often in a register largely ignored" (2015: 274 - 275).

\section{Geopolitical subjectivity}

Clearly, the Geoweb is a geopolitical phenomenon. Ontologically, a geopolitical phenomenon holds geopolitical value that can be exploited for some geopolitical purpose. Here, we will discuss the geopolitical use of the Geoweb or, in other words, its geopolitical subjectivity.

Popular Geoweb services are (almost) always designed to be easily accessible and free of charge. This, of course, is true of the Google Geoweb and of OSM. The idea of the Geoweb is therefore in line with that of "net neutrality" - the notion that all internet content should be processed in the same manner without discrimination (Crampton 2009a: 96). The concept of net neutrality is relevant in considering the correlation between general Internet accessibility, access to and participation in the Geoweb, and the geopolitical subjectivity of the Geoweb. Since not all information is equally accessible, it is reasonable to argue that societies in which more individuals can access the Internet and have enough knowledge to use and participate in the Geoweb will see more of its geopolitical benefits. And while technology has made accessing the Geoweb quite simple (through a basic Internet-connected smartphone), such access remains uneven across the globe, as a matter of means, infrastructure, and/or knowledge.

OSM participation data offers a good illustration of this uneven distribution. Pascal Neis, a German programmer and digital geo-data enthusiast, has developed OSMstats - an independent, free-of-charge website that, as the name suggests, automatically generates OSM statistics - offering daily user participation data (number of users and changes made), presented 
by country of origin. Analysing this data, it becomes apparent that the largest share in participation comes from the same handful of countries: Germany (almost always first), the United States (almost always second), Russia, France, Italy, the United Kingdom, and Poland. There is no reason to believe participating users from these countries are anything but independent mapping enthusiasts, but their personal geopolitical culture and heritage inevitably produce a specific geopolitical subjectivity. It is thus safe to conclude that OSM has a general inclination toward the geopolitical traditions (interests, and even agendas) of those countries. This is in fact reflected in OSM's wiki-based participatory system. Just as Wikipedia editors can change other users' article content, participating users in OSM can change other users' cartographic inputs. This leads to persistent online quarrels that manifest in cartographic data and political labels that are constantly changing for disputed areas ${ }^{7}$.

We were unable to find user participation statistics for the Google Geoweb, but as noted earlier, participation is not at the core of its functioning. Its cartographic political labels (borders, demarcations, names, etc.) are determined by Google's team of experts. Because Google is shareholderowned, it should be an independent and objective party in this process; however, it is a bit more complicated than that. Though Google operates globally and has shareholders on every continent, the company was founded in the US and is bound by American laws and regulations, which influence the geopolitical narrative of its Geoweb. What's more, Google Earth satellite imagery is limited by US law, as image providers must follow strict distribution and publishing regulations (Kumar 2010: 170). For example, the government can "put any area off limits in the interests of national security" and very high-resolution images are subject to a 24hour delay before publishing ${ }^{8}$ (Hafner and Rai 2005 by ibidem).

In discussing geopolitical subjectivity in the Google Geoweb, it is important to consider the notion of geopolitical scalability. Defarges highlighted

7 It should be noted that OSM has mechanisms in place to enforce objective mapping. In our opinion, there are two problems with this validation process: (i) it relies on users to find problematic inputs and fix them, and (ii) what one user sees as true may not be true to another user (truth is quite relative). More about OSM input validation can be found at the MapBox website on the page entitled "Validating OpenStreetMap."

8 Geopolitical influence is plainly apparent in some of these laws: one of those mentioned by Kumar proscribes that "images of Israel shot by American-licensed commercial satellites [are to] be made available only at a relatively low resolution" (2010: 170). 
the difference between "micro-geopolitics" and "classical geopolitics", wherein micro-geopolitics involves "micro-entities" like regions, cities, and multinational companies (2006: 136 and 137). Thus, Google should operate within the realm of micro-geopolitics; and yet sometimes, this rule doesn't hold, as Google's utilization of satellite imagery and the political labels in its Geoweb have become points of dispute between Google and several nation-states. Kumar described some publicly known disputes in a 2010 paper, noting that they emerged as soon as Google introduced its Geoweb services. The first case came to the fore in August 2005 when the Australian Nuclear Science and Technology Organization (ANSTO) called for censorship of images of its nuclear reactors. One week later, two Dutch MPs wrote a letter to their government outlining concerns over the possible harm done to national security by these Google services. In both instances, Kumar notes, Google pointed out that the images could be obtained from other services as well and asserted that the benefits "far outweigh any negatives" (2010: 160 -161).

But not all disputes were set aside so easily, and in the context of arguments made in this paper, a particularly good case study is what Kumar describes as Google's "showdown with India" - which lasted over a year and a half and involved threats and "publicly expressed frustration." The dispute started in September 2005 after a report by the Times of India that images of Indian security sites were available on the web. It did not take long for the dispute to grow to encompass the depiction of India's borders in disputed areas. Kumar notes that even Abdul Kalam, then President of India, got personally involved; and as a result, the Indian Embassy in the US received instructions to negotiate directly with Google. But Google ignored voices from India until an early 2007 visit there by Google Vice President Vinton Cerf, which finally ended the dispute after the company succumbed to India's demands. Google released a statement disclosing that images of disputed sites would be blurred and that, in labeling parts of Indian borders, variations would be marked (2010: 162-164).

One more interesting case should also be mentioned here, at least as a side note. In January 2013, Google executive Eric Schmidt traveled to North Korea ${ }^{10}$. This was quite an accomplishment given that the country's

9 In this particular instance, Defarges uses "classical" in opposition to "micro," regarding only scalability.

10 Schmidt was the Executive Chairman of Google. At the time of this writing, he holds the same position at Alphabet, 
most distinguishing attribute is its inaccessibility. Interestingly, at almost the same time, Western media reported that Google Earth users could view secret camps and prisons inside North Korea. Although the imagery was of a lower quality than that received by human rights activists via Digital Globe (a commercial satellite image provider), activists nonetheless found these images very useful (Eckert 2013).

These cases and more make it clear that Google cannot be confined within Defarges' framework of micro-geopolitical scale. Google, a nonstate actor, pushes nation-states to bypass the nation-state structure and give equal accommodations to a non-state actor, thereby ceding their national sovereignty (Kumar 2010: 169). Google has thus transcended micro-geopolitics and has positioned itself within Defarges' "classical geopolitics." It has also developed its own geopolitical subjectivity, despite an obvious inclination toward the geopolitical culture and traditions of the US. In this way, and in great part due to its Geoweb, Google can be described not only as a geopolitical phenomenon but as geopolitical agent or subject as well.

\section{Conclusion}

As Dodds explains, maps do not represent a neutral or transparent reality (Dodds 2007: 120). Still, any number of disciplines, and contemporary geopolitical theory among them, are touched by cartography. And since space is the ontological core of geopolitics, maps - these unobjective depictions of space - can be viewed as geopolitical statements. Modern, computer-aided space representation is no exception to this.

Indeed, digital cartography, the "new spatial media," is burdened by the same geopolitical properties as traditional maps: ethnocentricity and hierarchical space representation. And these "new" maps also reflect early 20th century geopolitische karte. Yet, digital cartography has its very own geopolitical narratives; Gerlach's "minor geopolitics" is one

Inc., which became Google's parent company after a 2015 reorganization. 
example, but there are many others, each distinct within the discourse of geopolitical subjectivity on the Geoweb.

The Google Geoweb specifically is confined by American laws and regulations, both because its satellite imagery providers are largely USbased and must abide by strict regulations on image publication designed to align with that country's national interests, and because the company itself was founded in the US and is compulsory to American markets, laws, and practices. The Google Geoweb is thus unable to guarantee its objectivity in the process of Geoweb content production, especially regarding access to satellite imagery without discrimination, regardless of the place depicted, and the determination of political labels. Its geopolitical subjectivity is tinged by American influence.

Both of the Geoweb services we used as the empirical foundation for this paper are propped up by user participation. As discussed, in the Google Geoweb, this is manifested only in add-on creation; whereas, OSM is built and functions entirely on the power of crowdsourcing. The collective nature of these projects, even in varying degrees, facilitates forms of geopolitical entanglement not seen in traditional cartography, as every participating user invests in their own geopolitical culture. In the Google Geoweb, this creates a cacophony of cartographically expressed political opinions, ideas, and theories. In OSM's base map, it results in constant changes based on various particular understandings of the features of political space, mostly in contested regions and areas.

As a company, Google should belong to the geopolitical space Defarges called micro-geopolitics, reflecting geopolitical scalability. Yet, largely due to its Geoweb, the company has engaged in disputes with several nation-states and its key personnel have had official and unofficial contact with governmental bodies. Google has thereby transcended the realm of micro-geopolitics and has obtained a geopolitical subjectivity per se.

This paper is a part of the discourse on the social and political impact of digital representations of space. The aim of this research was to explore how geopolitical narratives in cartography are reflected in the Geoweb. In other words, to examine the geopolitics-Geoweb relationship - how geopolitics influences the Geoweb and vice versa. As we have shown, all 
the geopolitical properties of traditional cartography are applicable to digital mapping, and new ones have developed. Moreover, geopolitical agents have gained a new tool and new subjects have been introduced. We believe this contribution is useful to burgeoning discourse in this field.

\section{References}

Agnew, J., 2003. Geopolitics: Re-visioning world politics Second edition. London/New York: Routledge.

Boria, E., 2008. Geopolitical Maps: A Sketch History of a Neglected Trend in Cartography. Geopolitics, 13(2): 278-308.

Caquard, S., 2011. Cartography I: Mapping narrative cartography. Progress in Human Geography. 37(1): 135-144.

Countries, OSMstats. Available at: http://osmstats.neis-one. org/?item=countries [accessed 12 April 2017].

Crampton, J. W., 2009a. Cartography: maps 2.0. Progress in Human Geography 33(1): 91-100.

Crampton, J. W., 2009b. Cartography: performative, participatory, political. Progress in Human Geography. 33(6): 840-848.

Defarges, P. M., 2006. Geopolitički rječnik. Zagreb: CPI.

Dodds, K. and Atkinson, D., 2003. Preface. In: Dodds, K and Atkinson, D. Geopolitical traditions: A century of geopolitical thought. pp. xiv xvi.

Dodds, K., 2005. Global Geopolitics, a critical introduction. Essex: Pearson.

Dodds, K., 2007. Geopolitics, a very short introduction. Oxford: Oxford University Press.

Eckert, P., 2013. Google Earth helps put North Korea gulag system on the map. Reuters. [online]9 January. Available at: http://www.reuters. com/article/us-korea-north-google-idUSBRE9081CS20130110 
[accessed 16 April 2017].

EPSG: 3857, OpenStreetMap wiki. Available at: http://wiki.openstreetmap. org/wiki/EPSG:3857 [accessed 8 February 2017].

Farman, J., 2010. Mapping the digital empire: Google Earth and the process of postmodern cartography. New Media \& Society. 12(6): 869-888.

Gerlach, J., 2015. Editing worlds: participatory mapping and a minor geopolitics. Transactions of the Institute of British Geographers. 40(2): 273-286.

Gordon, E., 2007. Mapping Digital Networks: From cyberspace to Google. Information, Communication \& Society. 10(6): 885-901.

Harley, J. B., 1989. Deconstructing the Map. Cartographica. 26(2): 1-20.

Kumar, S., 2010. Google Earth and the nation state: Sovereignty in the age of new media. Global Media and Communication. 6(2): 154-176.

Lee, M., 2010. A Political Economic Critique of Google Maps and Google Earth. Information, Communication \& Society. 13(6): 909-928.

Offen, K., 2012. Historical geography II: Digital imaginations. Progress in Human Geography. 37(4): 564-577.

Parsons, E., 2013. The Map of the Future May Not Be a Map! The Cartographic Journal. 50(2): 182-186.

Plantin, J.; Lagoze, C.; Edwards, P.N. and Sandvig, C., 2016. Infrastructure studies meet platform studies in the age of Google and Facebook. New Media \& Society. 1-18.

Program Corona, Mission and Spacecraft Library - NASA JPL. Available at: http://space.jpl.nasa.gov/msl/Programs/corona.html [accessed 8 February 2017].

Stats, OpenStreetMap wiki. Available at: http://wiki.openstreetmap.org/ wiki/Stats [accessed 9 February 2017].

Validating OpenStreetMap, MapBox. Available at: https://www.mapbox. com/mapping/validating-osm/ [accessed 6 May 2017].

Why NATO isn't Obsolete, Google Maps Mania, [online] 16 January 2017. 
Available at: http://googlemapsmania.blogspot.ba/2017/01/whyNato-isn't-obsolete.html [accessed 5 March 2017].

Yeo, S., 2016. Geopolitics of search: Google versus China? Media, Culture \& Society. 38(4): 591-605.

Sead Turčalo (sturcalo@gmail.com) is Assistant Professor at the Faculty of Political Sciences, University of Sarajevo. Sead lectures on geopolitics, international security, and international conflict management; and, along with these topics, also researches state-building, violent radicalization and other international security issues.

Ado Kulović (a.kulovic@gmx.com; ado@cmp.ba) is a researcher from Sarajevo. His research interests are mostly geopolitics and geopolitical theory with emphasize on the spatial particularities of the World Sea. He has published several original scientific and professional papers in Bosnia and Herzegovina and Croatia. Ado was one of the founders of the Center for International Politics, which is an independent research facility from Sarajevo. He has earned his BA and MA at the Faculty of Political Science, University of Sarajevo and is currently the PhD candidate in the field of Political Science at the University of Vienna. 